\title{
CONGENITAL LACRIMAL FISTULA
}

\author{
R. A .N. WELHAM, A. K. BATES, G. O. STASIOR \\ London
}

\begin{abstract}
SUMMARY
Congenital lacrimal fistula is a rare developmental condition. Patients may be asymptomatic or have tearing from the fistula, the eye or both. The clinical features of 60 patients are reviewed, including presenting symptoms, presence of other lacrimal or systemic anomalies and morphology of the fistula. A detailed description is given of the surgical technique utilised in patients whose symptoms warrant surgical intervention. The results of this technique are presented and alternative treatment strategies are discussed. Theories of the aetiology of the condition are reviewed and morphological and histological evidence is presented to support our belief that congenital lacrimal fistulae represent aberrant canaliculi.
\end{abstract}

Congenital lacrimal fistula was first described by Rasor $^{1}$ in 1675. Von Ammon ${ }^{2}$ reported a case in 1841 and there have been numerous reports in the literature since. We reported our experience of this condition some years ago, ${ }^{3}$ but since then differing views have been expressed regarding the optimal management of this condition and our experience has greatly increased.

Congenital lacrimal fistula is a rare developmental condition, estimated to occur in one in 2000 births, ${ }^{4}$ in which an epithelial lined tract connects the skin to the common canaliculus, lacrimal sac or nasolacrimal duct. In some cases the tract ends blindly in the sub-cutaneous tissue near the lacrimal sac. Most are unilateral and are located inferonasal to the medial canthus. ${ }^{3}$

These fistulae may be asymptomatic and therefore overlooked for some time after birth. In some cases it may be difficult to ascertain whether a fistula has been present since birth and remained unnoticed or has arisen later as a result of infection or surgical intervention. Symptoms may be of tearing from the ostium of the fistula or from the eye or both depending on whether there is also nasolacrimal obstruction.

\section{METHODS}

We reviewed the notes of all patients attending the Lac-

From: Lacrimal Clinic, Moorfields Eye Hospital, City Road, London. Correspondence to: A. K. Bates, Moorfields Eye Hospital, City Road, London EC1V 2PD. rimal Clinic at Moorfields Eye Hospital with the diagnosis of congenital lacrimal fistula between 1972 and 1990. All surgery was performed or supervised by one person (RANW). Detailed outcome of surgery was assessed objectively for the presence or absence of tearing from the eye or lacrimal fistula after the polyethylene tubing had been removed. This information was obtained by primary dye testing or the objective presence or absence of tearing as determined by the parents and ophthalmologist.

\section{Surgical Technique}

A vertical dacryocystorhinostomy incision was made $8 \mathrm{~mm}$ medial to the inner canthus, medial to the external opening of the fistula. Probes were placed in the superior and inferior canaliculus and fistula tract. The skin edges were retracted and the anterior crus of the medial palpebral tendon was divided near its bony insertion. Dissection in the plane between the anterior surface of the lacrimal sac and the deep aspect of the divided tendon allowed the common canaliculus to be identified and isolated and also the deep origin of the fistula. The periosteum was then seperated from the lacrimal bone and a large osteotomy performed. The tear sac was opened along its medial wall forming anterior and posterior mucosal flaps. The internal common punctum was then carefully inspected with manipulation of the probes in the canaliculi and fistula to define precisely the anatomical arrangement. The canaliculi were then intubated with polyethylene tubing. Anterior and posterior nasal mucosal flaps were then made opposite those in the lacrimal sac. The posterior flaps were sutured with interrupted $6-0$ Vicryl. A skin incision was then made from the external opening of the lacrimal fistula to the middle of the vertical dacryocystorhinostomy incision forming a "T". The fistula tract was then seperated from the surrounding tissues. A purse string absorbable $6-0$ suture was placed around the deep origin of the fistula and the tract was excised. The anterior flaps of lacrimal sac and nasal mucosa were closed and the canalicular tubing was tied and left in the nasal cavity. The medial palpebral tendon was reapproximated and the skin closed. The tubing was left in situ for three months. 


\section{RESULTS}

\section{Non-Surgical Cases}

Our review of clinical records found 25 patients with congenital lacrimal fistulas who did not undergo surgery after initial evaluation. Of these, 16 patients were fully evaluated, where necessary under anaesthesia, with syringing, probing and dacryocystography. The remainder had very minor or no symptoms and so were simply kept under observation.

This group had 14 females and 11 males. In 16 cases the fistula was on the right side, in seven it was on the left and in two cases it was bilateral. The mean age at onset of symptoms was 3.85 years (range birth -19 years). In five patients who were asymptomatic the fistula was noted at mean age 13.63 years (range $2-42$ years).

A positive family history of lacrimal disorders was obtained in three patients. One patient's mother was examined and found to have a fistula of which she was unaware, she was asymptomatic. One patient had a brother who had undergone lacrimal surgery for nasolacrimal duct obstruction with mucocoele. The father of one patient with bilateral canalicular atresia in addition to congenital fistula, also had bilateral canalicular atresia but without fistula.

At dacryocystography the fistula was found to take origin from the common canaliculus in seven cases (Fig 1), in nine other cases it was not possible positively to identify the origin of the fistula radiologically because of inadequate definition. Of the 16 patients seven were found to have nasolacrimal duct obstruction with mucocoele, five on the side of the fistula and two on the opposite side.

These patients had a number of other lacrimal anomalies. In two patients there was bilateral upper and lower canalicular agenesis, in one with total agenesis of the lacrimal system on the side with the fistula except for a tiny sac remnant. Two patients had an absent upper punctum on the side with the fistula, in one case with absent upper canaliculus as well and a duplicated lower canaliculus.

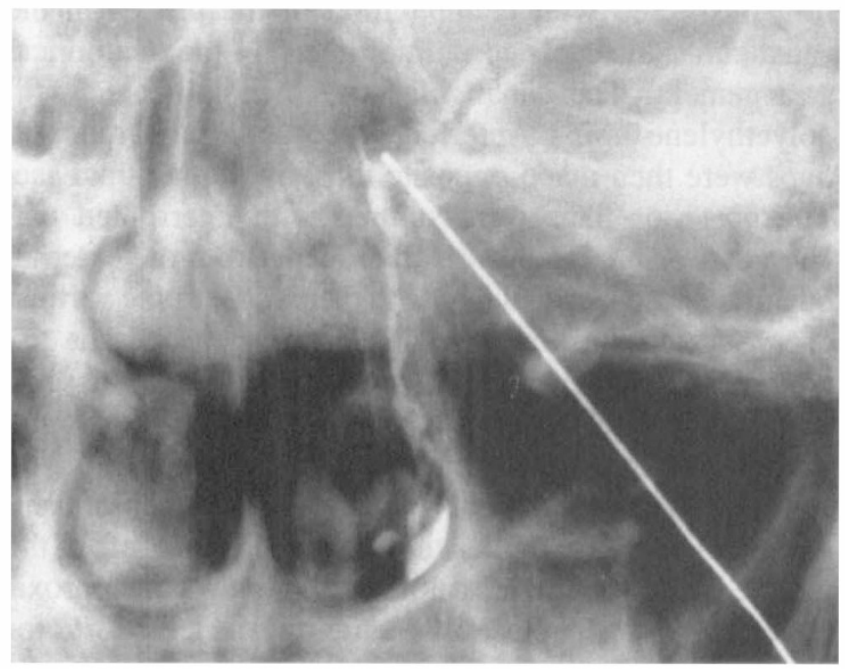

Fig. 1. Dacryocystogram with probe placed in fistula tract to show origin of fistula from common canaliculus.
Three patients had nasolacrimal duct obstruction on the other side. One patient had an accessory lower punctum on the same side as the fistula and also a diverticulum of the lacrimal sac. Thus in seven of the 25 (28\%) patients there was an associated lacrimal abnormality, excluding nasolacrimal duct obstruction on the same side as the fistula.

Two of the patients had Downs syndrome, two had strabismus, one had a tracheo-oesophageal fistula and one had a pre-auricular dimple on the opposite side to the fistula.

At presentation five of the patients were completely asymptomatic and had simply noted the presence of the fistula. In the remaining 20 patients (22 eyes) epiphora was the most common complaint, in two cases from the fistula, three cases from the eye and in 10 cases from both. Mucus discharge was the most prominent symptom in seven; from the eye in three and from the fistula and eye in four cases. One patient as well as discharge had several episodes of marked inflammation of the fistula. Thus of 22 symptomatic eyes with fistulae 16 had watering or discharge from the fistula and one had fistulitis.

\section{Surgical Cases}

Thirty-five patients underwent 38 operations for congenital lacrimal fistulae (three bilateral fistulae). A further seven patients with nine fistulae (two bilateral) who had previously had unsuccessful operations elsewhere were also treated surgically. Thus in total 42 patients had surgery for 47 fistulae. Twenty-six were male and 16 female. The fistula was on the right side in 20 cases, the left in 17 and five were bilateral.

In 29 of these patients symptoms commenced at birth (69\%) with mean age 3.3 years (range birth -41 years). None of the patients in this group were asymptomatic at presentation, although symptoms were not always directly attributable to the fistula itself. No patient in this. group gave any family history of lacrimal abnormalities.

In the seven patients who had previously undergone unsuccessful surgery, three had excision of fistula, two had dacryocystectomy and two had drainage of acute dacryocystitis. Four of these patients had been probed and syringed on multiple occasions (17 times in one case). Ten of the patients without previous surgical treatment had also undergone unsuccessful probing and syringing of the nasolacrimal duct.

Many of these patients had associated lacrimal anomalies. Five had fistulae on the other side. Partial or complete nasolacrimal obstruction was present on the same side as the fistula in 13 cases and on the opposite side in 4 cases (two bilateral).

Mucocoeles were present on the same side as the fistula in 14 cases and on the other side in three cases(two bilateral). Canalicular agenesis was present in six cases: affecting the upper in four, the lower in one on the side of the fistula and in one case there was bilateral upper canalicular agenesis. Upper and lower punctal agenesis was present in two patients, on both sides in one case and on the side opposite the fistula in the other. Thus $26 \%$ of patients had partial or complete nasolacrimal obstruction associated 

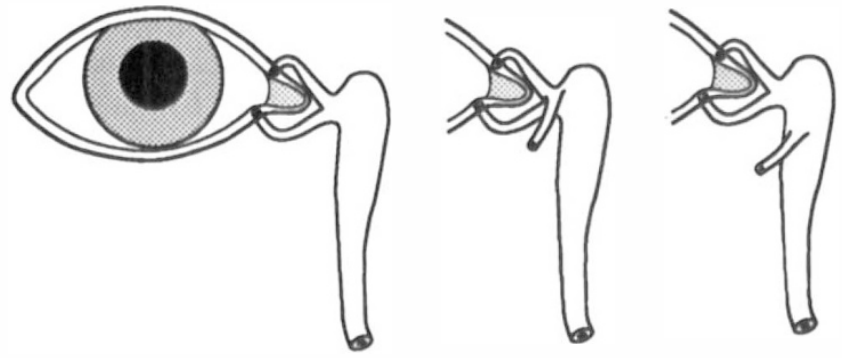

Fig. 2. Diagramatic representation of normal anatomy (left), fistula arising from the common canaliculus (centre) and from the lacrimal sac (right).

with a fistula and $29 \%$ of patients had a lacrimal anomaly on the opposite side to the fistula.

Some of the patients had non-lacrimal anomalies, four had either a pre-auricular fistula or dimple and three strabismus. All patients had epiphora from the eye and most also from the fistula. Six had episodes of acute dacryocystitis and three recurrent fistulitis.

Forty-two of the patients had fistula excision via a T-incision combined with dacryocystorhinostomy and intubation of the nasolacrimal system. Two patients had minimal syptoms from the fistula itself and so had dacryocystorhinostomy and intubation alone. Three patients had fistulectomy alone for recurrent fistulitis in the absence of any other lacrimal symptoms.

At surgery the fistula was found to take origin from the lacrimal sac in 11 cases and from the common canaliculus in 34 cases (Fig 2). Nine of the 11 cases in which the fistula arose from the sac had undergone previous surgery. In one case the fistula arose from the sac adjacent to the internal opening of the common canaliculus; in one there was a double fistula to the common canaliculus. The external openings of the fistulae originating from the common canaliculus were medial and inferior to the inner canthus with two exceptions. In these the fistula originated in the skin of the medial canthal angle adjacent to the canthus. Of the fistulae originating from the lacrimal sac, eight had external openings in the same position as those fistulae arising from the common canaliculus and two opened on the skin more inferiorly. The remaining fistula which originated from the fundus of the sac opened slightly above the medial palpebral ligament.

Follow up of three months or longer was available in all cases with mean eight months and range 3-96 months. Thirty-one patients were entirely symptom free at final follow up with no recurrence of the fistula and with a positive dye test. Two patients had recurrence of a slight dimple at the site of the fistula but it remained closed. One patient who was noted to have a stricture at the distal end of the common canaliculus at surgery had recurrence of tearing at four months post-operatively from the eye and is awaiting insertion of a Lester Jones tube.

One patient who had previously undergone surgery elsewhere for drainage of a lacrimal abscess had recurrence of the fistula at four months after surgery, she only noticed tearing from the fistula on blowing her nose and was not keen for further treatment. Histology was avail- able in 17 of the patients who had not undergone any previous surgery. This showed the fistula to be lined with stratified squamous epithelium in 15 cases (Fig 3), in one case (originating from the lacrimal sac) with transitional epithelium and in one case granulomatous tissue. Histology in two of the patients who had previously undergone surgery showed transitional epithelium in one case and vascularised fibrous tissue in the other.

\section{DISCUSSION}

The nasolacrimal drainage system arises from a thickening of the surface ectoderm in the naso-optic fissure in the $7 \mathrm{~mm}$ or 32 day embryo. This thickened epithelium becomes buried in the mesenchyme between the lateral nasal and maxillary processes in the $12 \mathrm{~mm}, 42$ day embryo and separates from the surface. Caudal and cephalic extension of the cord then occurs, forming the nasolacrimal duct and canaliculi. Segmental canalisation of the solid epithelial cord begins in the 32- to $36 \mathrm{~mm}, 60$ day embryo, and the puncta are patent by the seventh fetal month. At birth or soon after, the nasolacrimal duct opens into the inferior meatus-of the nose. ${ }^{5,6}$

Jones and Wobig ${ }^{7}$ call the cord of cells that extends inward from the surface the lacrimal anlage. The nasal end of this anlage forms the sac from whose superior aspect the canaliculi bud and migrate towards the lids. Jones and Wobig propose that this anlage fails to involute in cases of congenital lacrimal fistula, and that the cord of cells proliferates and canalises, forming the fistula.

Others have implicated amniotic bands in the aetiology of the fistula ${ }^{5}$ but none of our patients had evidence of amniotic bands elsewhere and it seems unlikely that they would result in such a tiny and isolated abnormality.

We have previously proposed that a congenital lacrimal fistula is really an extra canaliculus. ${ }^{3}$ The evidence from this much larger series of patients supports this view. This aberrant canaliculus extends from the common canaliculus to the skin in the medial canthal area while the superior and inferior canaliculi are also being formed. The fact that there can be abnormal budding of the canaliculi was confirmed by the large number of canalicular abnormalities in both our non-surgical and surgical patients.

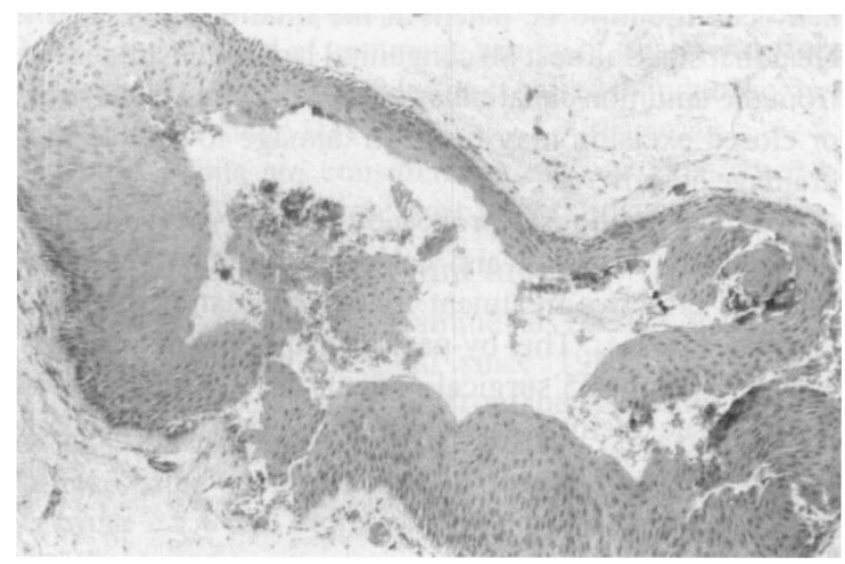

Fig. 3. Histology of fistula showing non-keratinising stratified squamous epithelium ( $H \&$ E stain). 
Histological evidence from this series also provides support for this theory with all the fistulae arising from the common canaliculus which were examined being lined by stratified squamous epithelium as in normal canaliculi. Thus it seems much more likely that such fistulae represent an outgrowth from the common canaliculus rather than an ingrowth from the surface. These findings are in contrast to those from patients with either a history of infection or inflammation, of previous (often multiple) surgical intervention or both. In such cases the fistula invariably arises from the lacrimal sac rather than from the common canaliculus, is not lined by stratified squamous epithelium and is rarely associated with other canalicular abnormalities.

Various authors have commented upon the hereditary pattern of lacrimal fistulas. Jones and Wobig ${ }^{7}$ presented a family in which bilateral lacrimal fistulae were present in four generations and followed an autosomal dominant inheritance pattern. Other authors since have also reported a high incidence of bilaterality of fistulae and have supported the view of an autosomal dominant inheritance pattern. ${ }^{4,89.10}$ In our 60 patients only five had bilateral fistulae and only one a family history of fistula (an asymptomatic mother who was noted to have a fistula only when she presented with her symptomatic child) although two other patients had family members with known lacrimal abnormalities, canalicular agenesis in one case and nasolacrimal obstruction in the other.

Systemic associations with fistula were not common, the only one which may be of significance was the presence of a pre-auricular dimple or fistula which was present in five patients. One patient with bilateral lacrimal fistulae also had a pre-auricular fistula which had been treated surgically ten years previously. He had a fraternal twin without lacrimal or auricular fistula. Others have reported the coexistence of lacrimal and auricular fistulae in family members. ${ }^{.1}$

The mode of treatment of lacrimal fistula remains controversial. In 1929 Levine $^{12}$ reported cauterising a lacrimal fistula in a seven year old boy. Unfortunately whilst this closed the fistula it resulted in considerable tearing which was considered surprising because the nasolacrimal duct had been found to be patent at the time of surgery. It is clear that since almost all congenital lacrimal fistulae arise from the common canaliculus simple probing, cauterising or closed excision may result in damage to the lacrimal drainage system.

We have previously proposed that dacryocystorhinostomy, fistula excision and canalicular intubation offers safe and effective treatment for symptomatic congenital lacrimal fistulas. ${ }^{3}$ This by-passes distal obstruction present in 13 of our 35 surgical patients (37\%). Opening the lacrimal sac facilitates surgical dissection and accurate ligation and removal of the fistula from its origin, minimising the risk of damage to the common canaliculus. In addition the incidence of recurrence of the fistula should be reduced because of the decreased resistance to outflow of tears effected by the dacryocystorhinostomy (DCR). Tears should therefore preferentially flow through the DCR rather than through the fistula; this is supported by the experience from this series where only one patient had recurrence of a symptomatic fistula. Intubation of the common canaliculus is recommended in all cases in which the common canaliculus has been manipulated and thus is much simpler and safer through a DCR.

Others in smaller series have reported successful management with simple fistula excision alone or combined with canalicular intubation in cases with nasolacrimal duct obstruction. ${ }^{13}$ However three of the patients in this series had been treated elsewhere by closed fistula excision alone and the fistula had recurred. We believe that closed fistula excision is more difficult and potentially damaging to the common canaliculus than an open approach where the anatomical relationships can be properly defined.

Key words: Congenital, Dacryocystorhinostomy, Fistula, Lacrimal.

\section{REFERENCES}

1 Rasor C, cited by Schirmer R: Graefe-Saemisch Handbuch der Augenheilkunde. Leipzig, Germany, Engelman, 1877 vol $81-58$.

2 Von Ammon FA: Klinische Darstellungen der Krankheiten und Bildungsfehler des Menschlichen Auges. Berlin, Germany, Reimer, 1841.

3 Welham RAN and Bergin DJ: Congenital Lacrimal Fistulas. Arch Ophthalmol 1985, 103: 545-8.

4 François J and Bacskulin: External congenital fistulae of the lacrimal sac. Ophthalmologica 1969, 159: 249-61.

5 Duke-Elder S: System of Ophthalmology. St Louis. CV Mosby and Co, 1963: Vol 3 241-6 and 936-7.

6 Cassady JV: Developmental anatomy of nasolacrimal duct. Arch Ophthalmol 1952, 47: 141-58.

7 Jones LT and Wobig JL: Surgery of the eyelids and lacrimal system. Birmingham, Ala. Aesculapius Publishing Co, 1976: 67, 167-173.

8 Sedan J: Canalicules lacrymaux surnumeraires et fistules congenitales du sac chez cinq membres d'une meme famille. Ann Oculist 1949, 182: 855-8.

9. Caillaud F: Fistule congenitale du sac lachrymal. Arch Ophthalmol 1906, 26: 167-70.

10. Penzani B: Su di un caso di fistolo lacrimale congenita. Ann Ottal 1952, 78: 255-8.

11. Mukherji R and Mukhopardy S: Congenital bilateral lacrimal and preauricular fistulae. Am J Ophthalmol 1972, 73: 595-6.

12. Levine J: Congenital fistula of the lacrimal sac. Am JOphthalmol 1929, 12: 745-6.

13. Birchansky LD, Nerad JA, Kersten RD, Kulwin DR: Management of congenital lacrimal sac fistula. Arch Ophthalmol 1990, 108: 388-90. 